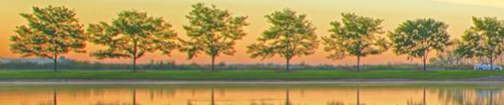

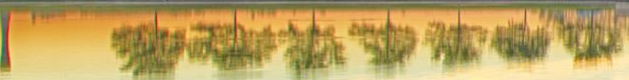

\section{Approaching scalable VQE of interacting bosons with NISQ devices}

Andy C. Y. Li

APS March Meeting 2020

3 March 2020
This document has been authored by Fermi Research Alliance, LLC under Contract No. DE-AC02-07CH11359 with the U.S. Department of Energy, Office of Science, Office of High Energy Physics. 


\section{Boson encoding by qubits}

Goal: encode a truncated boson Hilbert space in qubits

Position basis binary encoding

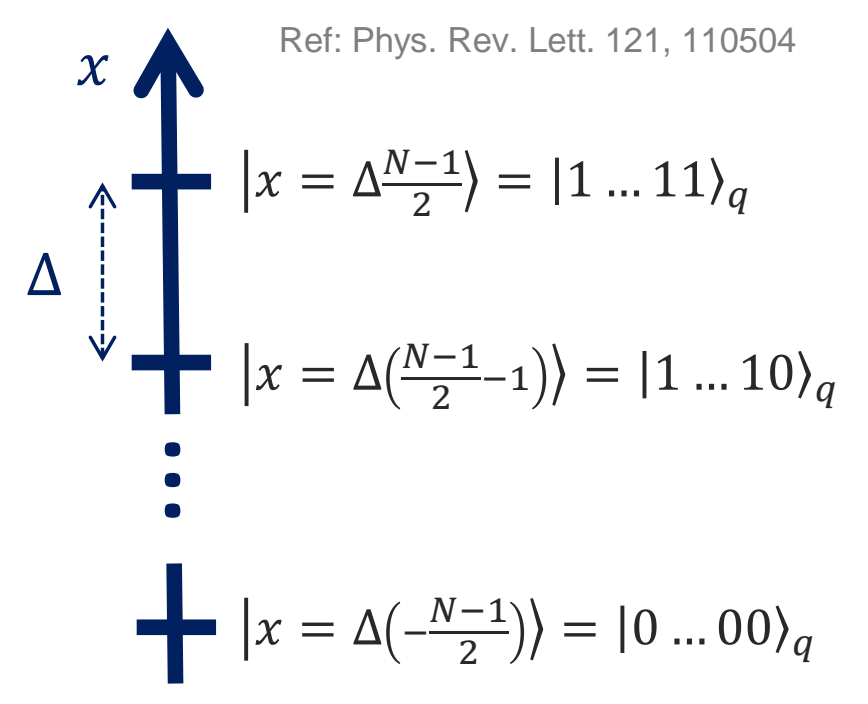

Number basis binary encoding

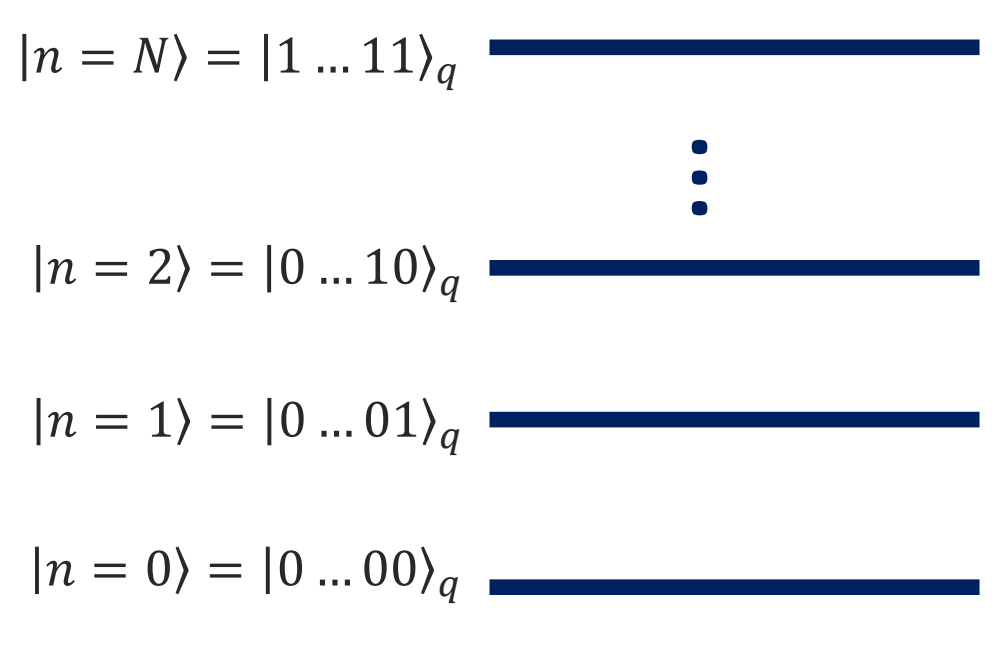




\section{Measuring expectation value with number-basis binary encoding}

(a)

$|\phi\rangle$

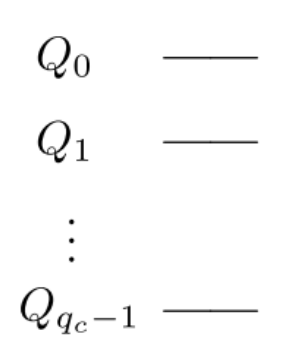

(b)

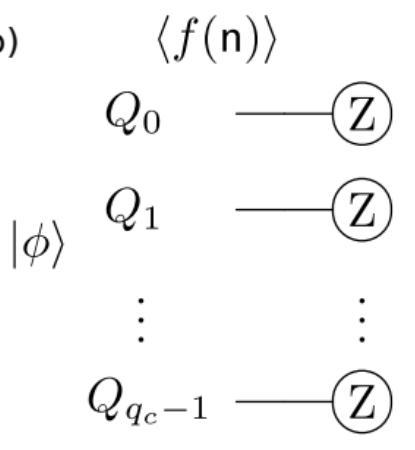

(c)

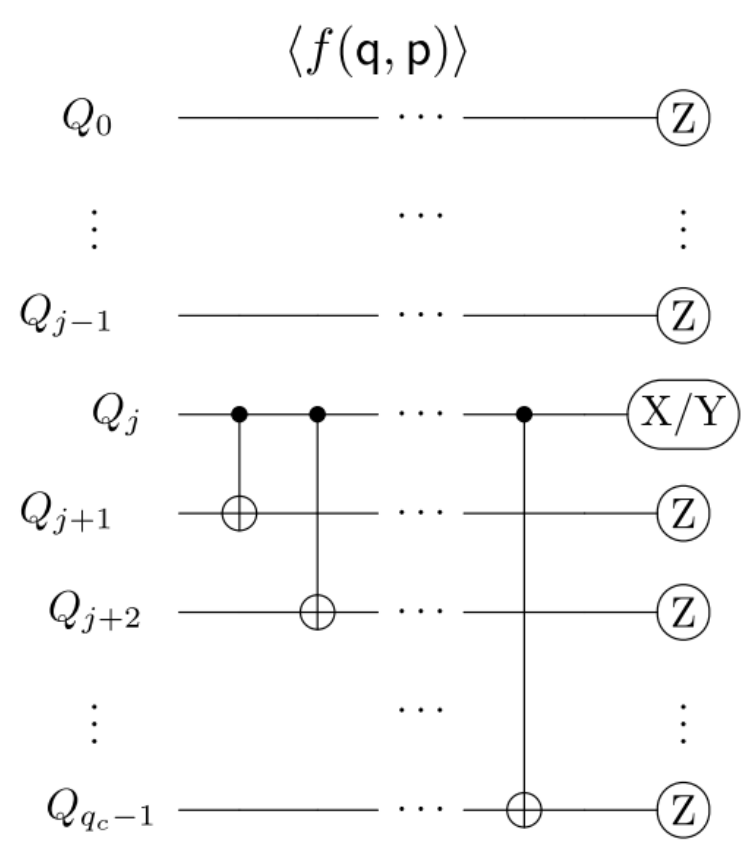

$n_{c}$ boson with $N_{I}$-mode interaction

\begin{tabular}{|c|c|c|}
\hline $\begin{array}{c}\text { Qubit count } q_{c} \\
\text { per boson } \\
\text { mode }\end{array}$ & $O\left(\log _{2} n_{c}\right)$ & $O\left(\log _{2} n_{c}\right)$ \\
\hline $\begin{array}{c}\text { Sampling } \\
\text { count }\end{array}$ & $O\left(q_{c}{ }^{\left.N_{I} \log _{2} \frac{n_{A}}{N_{I}}\right)}\right.$ & $O(1)$ \\
\hline $\begin{array}{c}\text { Pate } \\
\text { bate count }\end{array}$ & $O\left(N_{I} q_{c}\right)$ & $O\left(N_{I} q_{c}^{2}\right)$ or \\
\hline
\end{tabular}




\section{Proof-of-principle expt. - Rabi model using Rigetti's device}

TLS

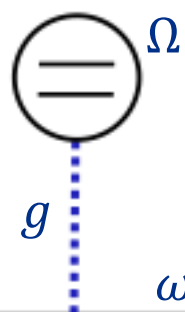

Rabi Hamiltonian: two-level system (TLS) coupled to a photon mode

$$
H=\omega a^{\dagger} a+\frac{\Omega}{2} \sigma_{z}+g\left(a^{\dagger}+a\right) \sigma_{x}
$$

Number-basis binary encoding:

Photon

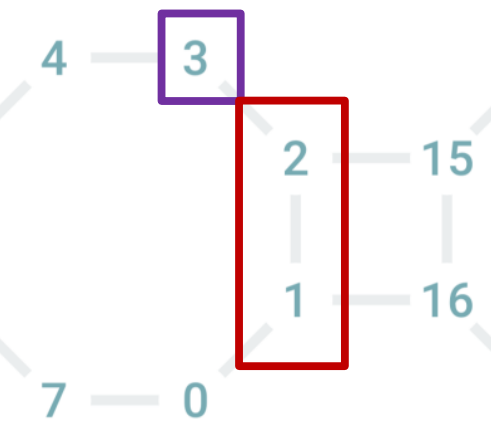

$14-13$

12

$$
\begin{aligned}
& \mathrm{R}_{\mathrm{Y}}\left(\theta_{0}\right)-\mathrm{R}_{\mathrm{Z}}\left(\theta_{1}\right) \cdot \mathrm{R}_{\mathrm{Y}}\left(\theta_{6}\right)-\mathrm{R}_{\mathrm{Z}}\left(\theta_{7}\right) \\
& \mathrm{R}_{\mathrm{Y}}\left(\theta_{2}\right)-\mathrm{R}_{\mathrm{Z}}\left(\theta_{3}\right) \cdot \mathrm{R}_{\mathrm{Y}}\left(\theta_{8}\right)-\mathrm{R}_{\mathrm{Z}}\left(\theta_{9}\right) \cdot \mathrm{R}_{\mathrm{Y}}\left(\theta_{10}\right) \mathrm{R}_{\mathrm{Z}}\left(\theta_{11}\right)-
\end{aligned}
$$

11

$$
\begin{aligned}
& |n=0\rangle=|00\rangle_{q}|n=1\rangle=|01\rangle_{q} \\
& |n=2\rangle=|10\rangle_{q}|n=3\rangle=|11\rangle_{q}
\end{aligned}
$$




\section{Hardware efficient trial state's ansatz}

1Q-gate layer

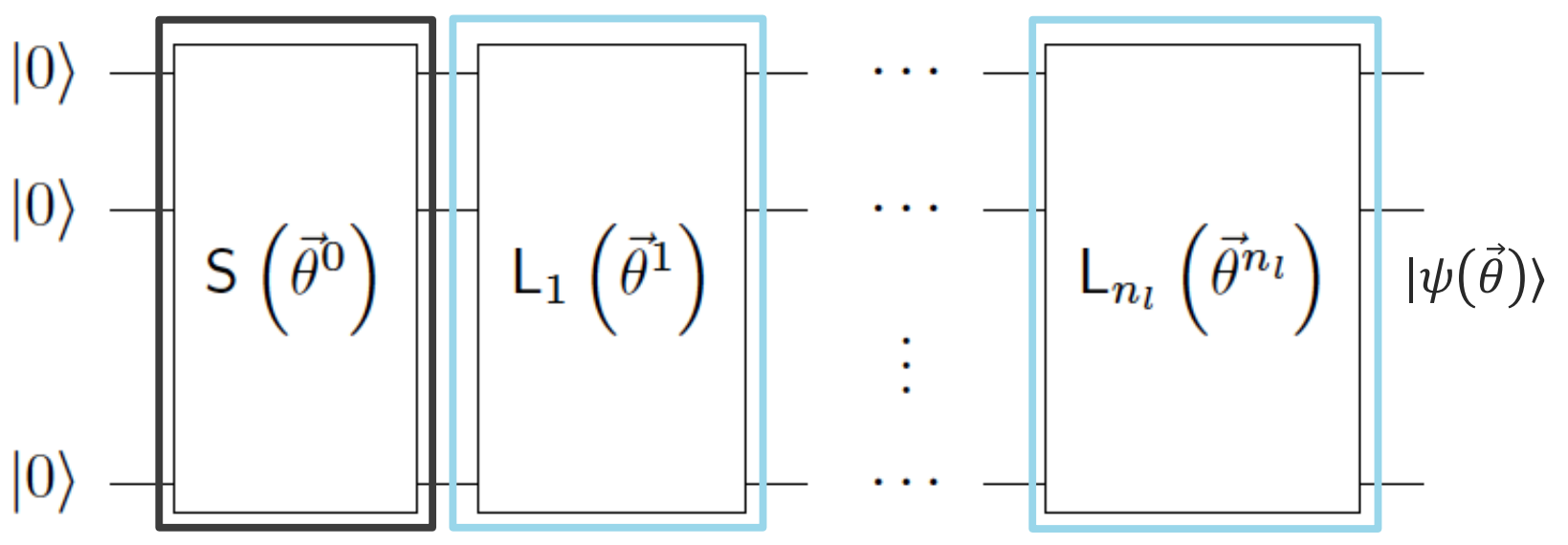

Ansatz consists only of native gates supported by the hardware e.g. $\mathrm{R}_{\mathrm{Y}}(\theta), \mathrm{R}_{\mathrm{Z}}(\theta)$ and $\mathrm{CZ}$ for Rigetti's devices

Example:

3 qubits with

1 entanglement layer

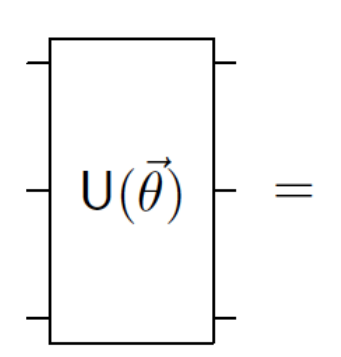

\begin{tabular}{|l|l|l|l|}
\hline $\mathrm{R}_{\mathrm{Y}}\left(\theta_{0}\right)$ & $\mathrm{R}_{\mathrm{Z}}\left(\theta_{1}\right)$ \\
\hline $\mathrm{R}_{\mathrm{Y}}\left(\theta_{2}\right)$ & $\mathrm{R}_{\mathrm{Z}}\left(\theta_{3}\right)$ \\
\hline $\mathrm{R}_{\mathrm{Y}}\left(\theta_{4}\right)$ & $\mathrm{R}_{\mathrm{Y}}\left(\theta_{5}\right)$ & $\mathrm{R}_{\mathrm{Y}}\left(\theta_{8}\right)$ & $\mathrm{R}_{\mathrm{Z}}\left(\theta_{7}\right)$ \\
\hline & & $\mathrm{R}_{\mathrm{Z}}\left(\theta_{9}\right)$ & \\
\hline & & $\mathrm{R}_{\mathrm{Y}}\left(\theta_{10}\right)$ & $\mathrm{R}_{\mathrm{Z}}\left(\theta_{11}\right)$ \\
\hline & & $\mathrm{R}_{\mathrm{Y}}\left(\theta_{12}\right)$ & $\mathrm{R}_{\mathrm{Z}}\left(\theta_{13}\right)$ \\
\hline
\end{tabular}




\section{Cost function for ground state \& excited states}

Ground-state cost function = trial state's energy

$$
C_{0}=\langle\psi(\vec{\theta})|H| \psi(\vec{\theta})\rangle
$$

Ground state: $\left|\psi_{0}\right\rangle=\operatorname{argmin} C_{0}$

$$
|\psi(\vec{\theta})\rangle
$$

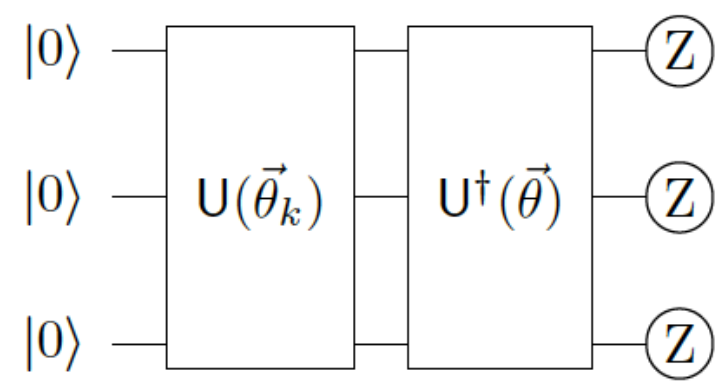

1st-excited state cost function: $C_{1}=\langle\psi(\vec{\theta})|H| \psi(\vec{\theta})\rangle+\underline{\left.\epsilon\left|\left\langle\psi_{0}\right| \psi(\vec{\theta})\right)\right|^{2}}$

1st-excited state: $\left|\psi_{1}\right\rangle=\operatorname{argmin} C_{1}$

Overlap with the ground state $|\psi(\vec{\theta})\rangle$

2nd-excited state cost function: $C_{2}=\langle\psi(\vec{\theta})|H| \psi(\vec{\theta})\rangle+\epsilon\left|\left\langle\psi_{0} \mid \psi(\vec{\theta})\right\rangle\right|^{2}+\epsilon\left|\left\langle\psi_{1} \mid \psi(\vec{\theta})\right\rangle\right|^{2}$ 


\section{Optimizers}

\begin{tabular}{|c|c|}
\hline Optimization algorithm & \\
\hline $\begin{array}{c}\text { Simultaneous Perturbation } \\
\text { Stochastic Approximation (SPSA) }\end{array}$ & Stochastic \\
\hline $\begin{array}{c}\text { Constrained Optimization BY Linear } \\
\text { Approximations (COBYLA) }\end{array}$ & Gradient-free \\
\hline $\begin{array}{c}\text { Bound Optimization BY Quadratic } \\
\text { Approximation (BOBYQA) }\end{array}$ & Gradient-free \\
\hline $\begin{array}{c}\text { Covariance Matrix Adaptation } \\
\text { Evolution Strategy (CMA-ES) }\end{array}$ & Gradient-free \\
\hline
\end{tabular}




\section{Optimizer with noisy device}

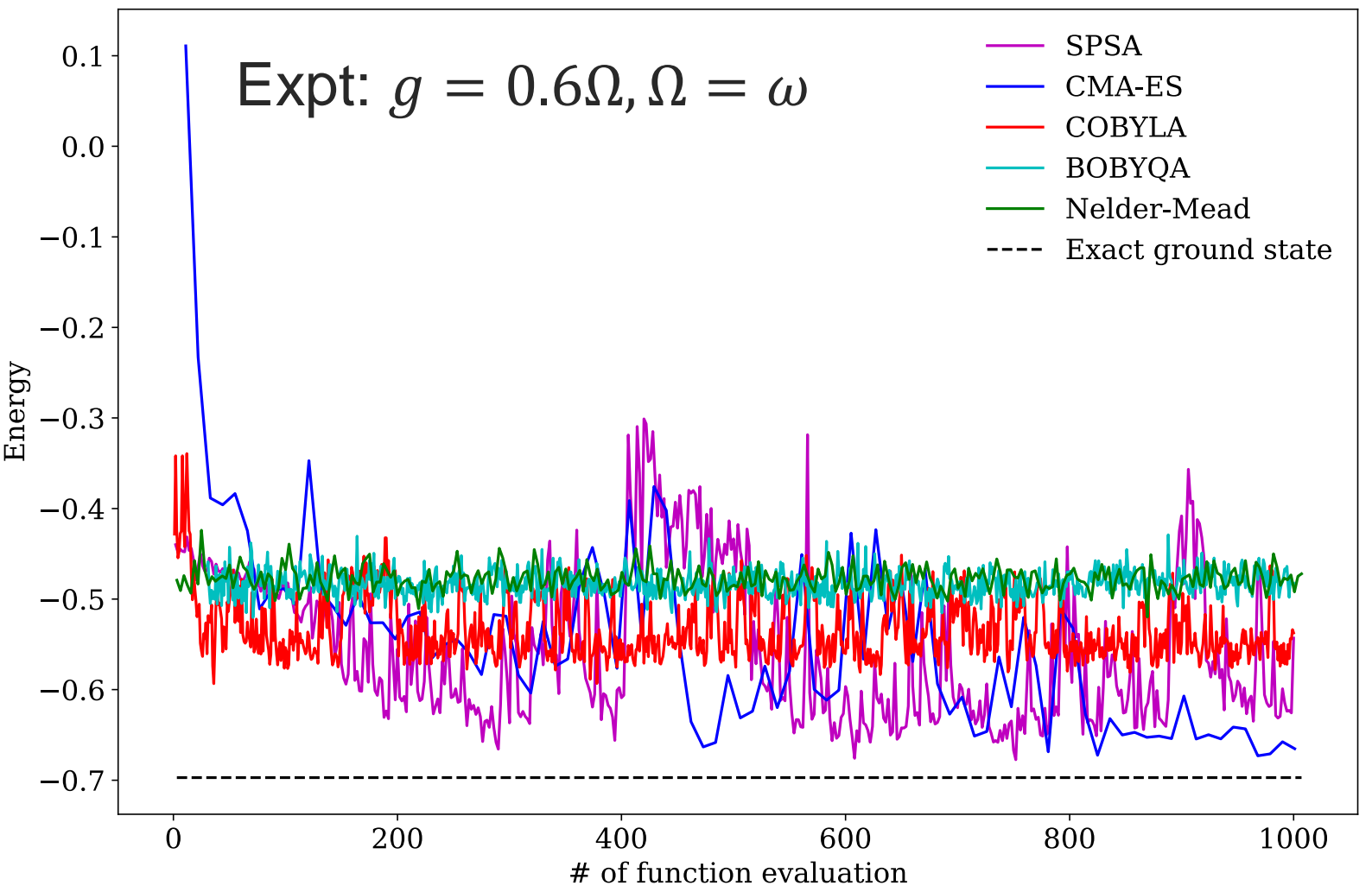

\section{Optimizer $\left|\boldsymbol{E}-\boldsymbol{E}_{\text {exact }}\right|$}

CMA-ES $\quad 0.062$

SPSA $\quad 0.099$

COBYLA $\quad 0.165$

BOBYQA $\quad 0.219$

Nelder-Mead $\quad 0.223$

- Stochastic algorithm $\checkmark$

- CMA-ES: slightly better 


\section{Summary}

- Scalable number-basis encoding scheme

- Proof-of-principle experiment of Rabi model

- 3-qubit implementation on Rigetti's device

- Ground state and 1st excited state

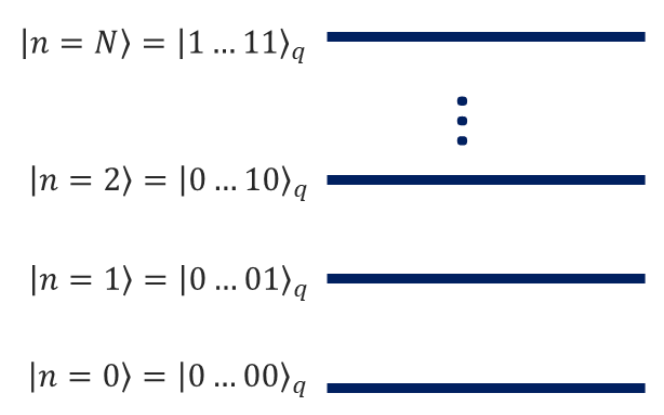

- Future works

- Trial state's ansatz

- Error mitigation techniques

- Lattice models: Rabi lattice, Holstein model...

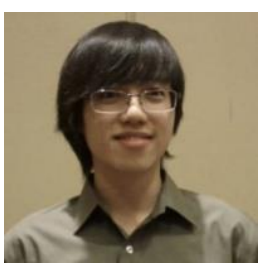

Andy C. Y. Li

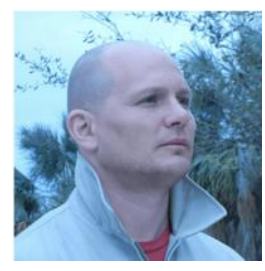

Alex Macridin

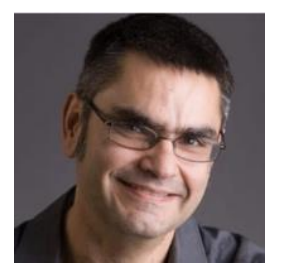

rigetti
葉Fermilab
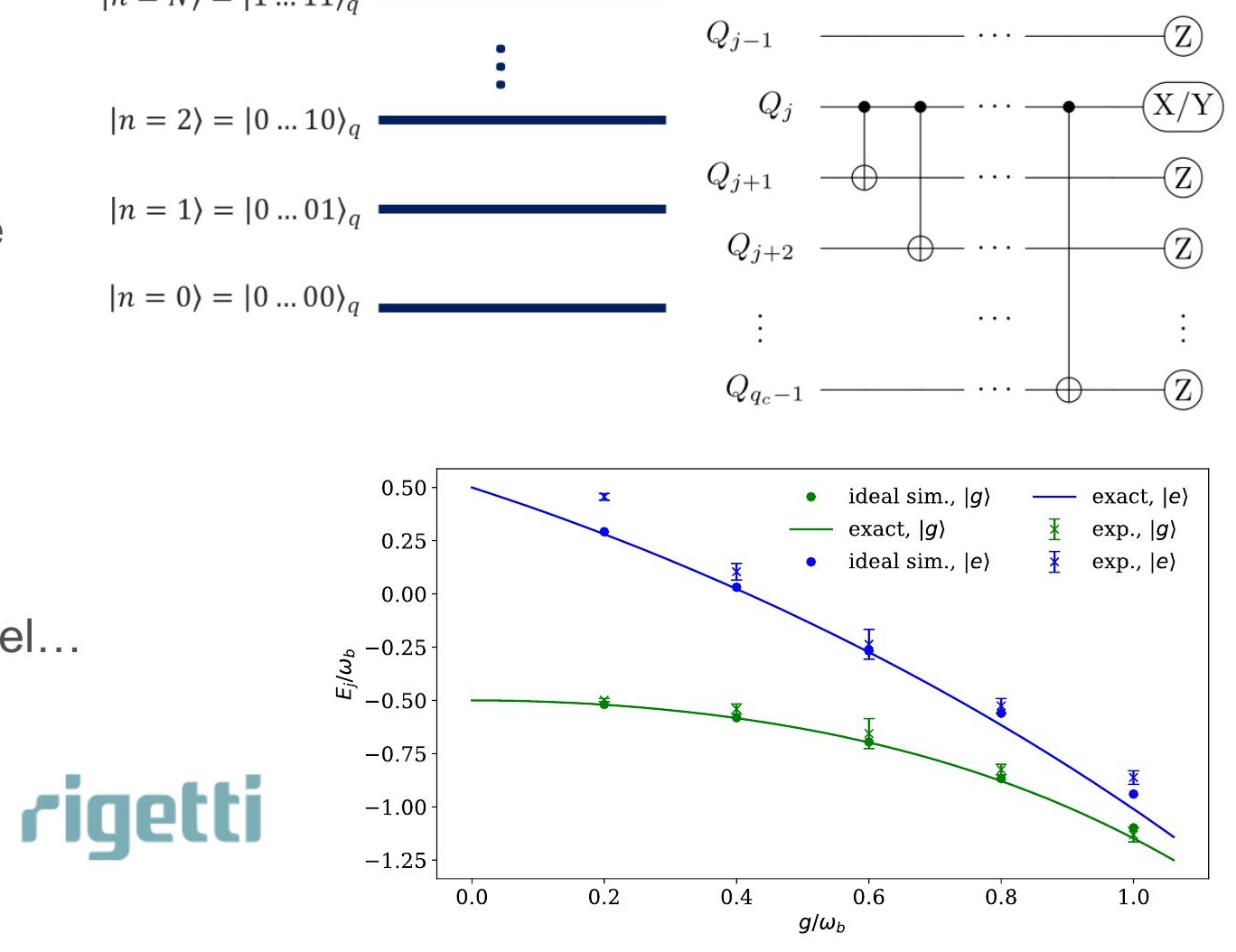